\title{
An unusual cause of acute pulmonary embolism: giant hepatic hemangioma
}

\author{
Hatice Duygu Bas MD, Supannee Rassameehiran MD, Kazim Baser MD, \\ Weeraporn Srisung MD, Mamoun Bashir MD, Tinsay Woreta MD, MPH
}

\begin{abstract}
Hemangiomas are the most common benign hepatic tumors and are usually asymptomatic. Lesions measuring more than $4 \mathrm{~cm}$ in diameter are known as "giant hemangiomas" and may cause various symptoms or complications depending on the size, the location, and the degree of compression of adjacent structures. Pulmonary embolism is a very rare complication of giant hepatic hemangiomas. In this case report, we describe a patient with acute pulmonary emboli, which presumably originated from laminar thrombi in the inferior vena cava caused by compression by giant hepatic hemangiomas.
\end{abstract}

Key words: giant hepatic hemangioma, acute pulmonary embolism, inferior vena cava thrombosis

\section{INTRODUCTION}

Giant hepatic hemangiomas, characterized as cavernous hemangiomas larger than $4 \mathrm{~cm}$ in diameter, may cause various symptoms or complications depending on the size, the location, and the degree of compression of adjacent structures. Pulmonary emboli occasionally arise from deep vein thrombosis (DVT) in less common locations, including the inferior vena cava (IVC). Inferior vena cava thrombosis is not a rare but is an under recognized entity which may result from external compression of the IVC by giant hepatic hemangiomas.

Corresponding author: Hatice Duygu Bas MD

Contact Information: H.Duygu.Baser@ttuhsc.edu DOI: 10.12746/swrccc2016.0415.205

\section{CASE}

A 53-year-old African American man presented with a 3-day history of worsening shortness of breath, pleuritic chest pain, and hemoptysis. On admission, the patient was hemodynamically stable, and his physical examination, including chest and cardiovascular exams, was within normal limits, except for diffuse abdominal tenderness and hepatomegaly. His past medical history was remarkable for hypertension. He drank alcohol daily and reported recreational drug abuse.

He reported no recent surgery or immobilization, prior venous thromboembolic event, or family history of hypercoagulability. Computed tomography (CT) angiogram of the chest showed bilateral segmental pulmonary emboli in the upper, right middle, and lower lobes. Hypercoagulability work up was negative, and color Doppler ultrasonography (US) did not reveal any DVT in lower extremity veins. 
Abdominal imaging studies were performed for the evaluation of the hepatomegaly and diffuse abdominal tenderness. Contrast-enhanced CT scanning of the abdomen revealed two large low density lesions in the liver, one in segment IV measuring approximately $15 \times 13 \mathrm{~cm}$ in size and another in segment VIII measuring $9 \times 14 \mathrm{~cm}$ in size. There was peripheral nodular "puddling" with enhancement slowly filling in toward the center, becoming increasingly isodense at the periphery on the delayed scan, suggestive of a giant cavernous hemangioma. There were two smaller lesions $(1.7 \times 1.6 \mathrm{~cm}$ and $3 \times 2.6 \mathrm{~cm}$ in size) with a similar enhancement pattern. The diagnosis was confirmed with magnetic resonance imaging (Figure 1). Abdominal CT also revealed low-density changes within hepatic hemangiomas on delayed phase imaging, possibly due to partial thrombotic changes in the hepatic hemangiomas. Hepatic Doppler US revealed a filling defect in the center of the retrohepatic inferior vena cava (IVC) with high blood velocities adjacent to the region, consistent with intraluminal thrombosis (Figure 2). Hepato-petal flow in the portal vein and flow in the hepatic artery and hepatic veins were normal.

Surgical resection did not seem possible in this case due to large and multiple lesions that occupied nearly all the liver. Long-term anticoagulation with warfarin was started.
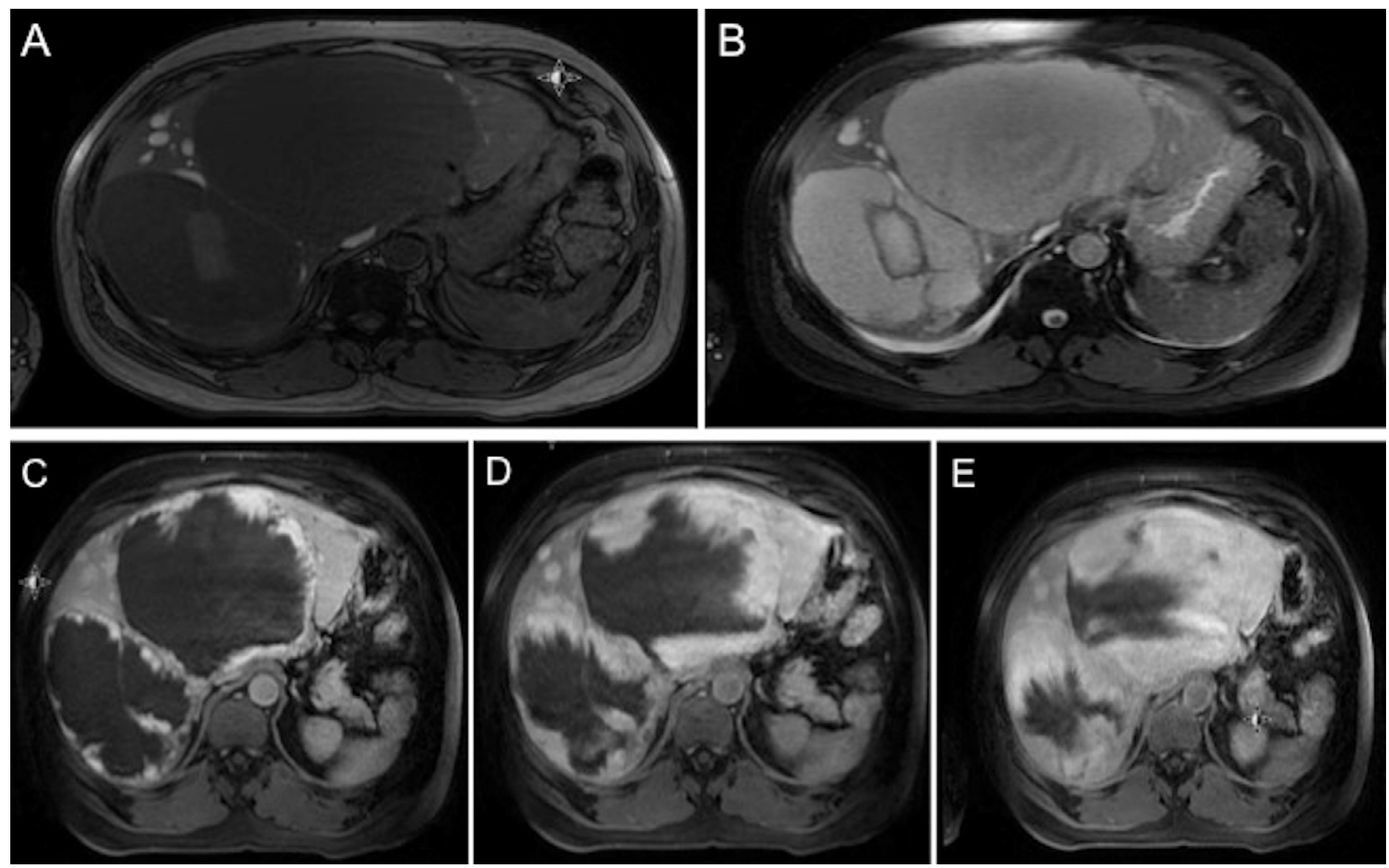

Figure 1: The lesions appear as hypointense masses on T1-weighted magnetic resonance imaging (MRI). B) The lesions appear as hyperintense masses on dual-echo T2-weighted MRI, C-D-E) Dynamic gadoliniumenhanced MRIs, demonstrating the progressive, centripetal contrast enhancement of hepatic hemangiomas. 

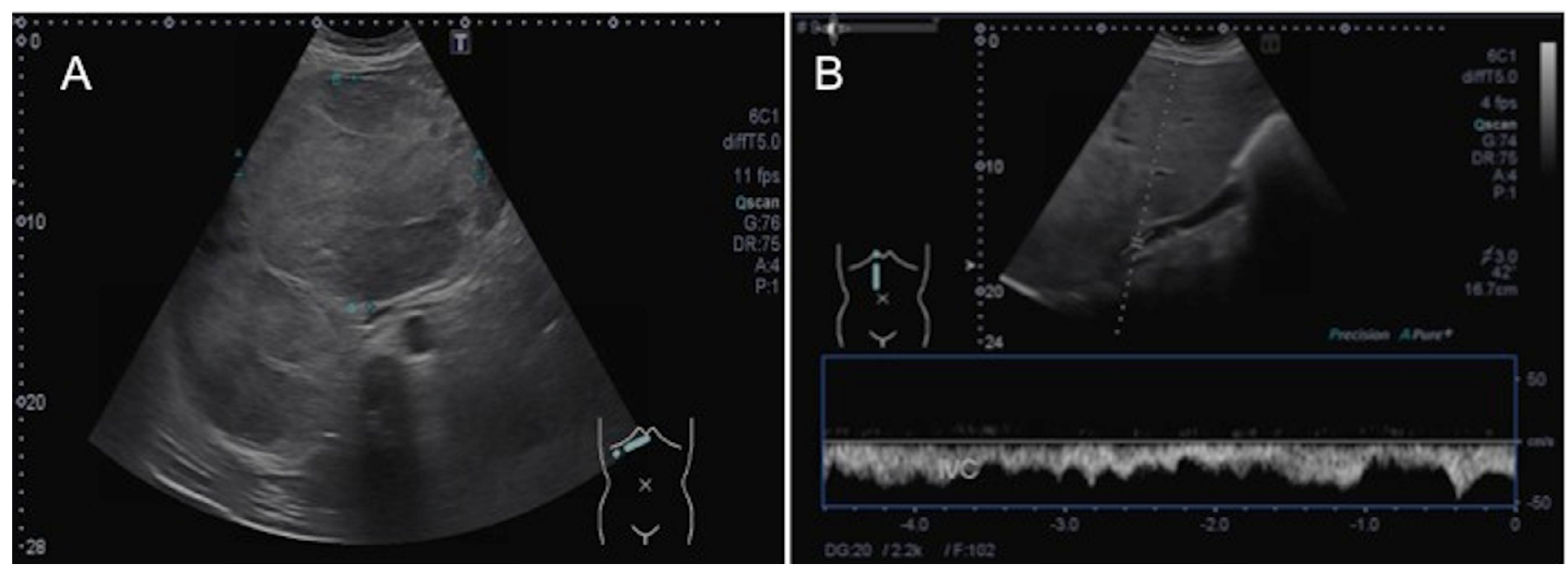

Figure 2: A) Abdominal US shows two large hyperechoic masses in liver and their close anatomic relationship with IVC. The largest hyperechoic mass measures $16 \mathrm{~cm}$ in greatest dimension located in the right hepatic lobe. B) Hepatic Doppler US reveals a filling defect in the center of the retrohepatic IVC with high blood velocities adjacent to the region.

\section{Discussion}

Cavernous hemangiomas are the most common primary benign liver neoplasm, with a prevalence ranging from $3 \%$ to $20 \%$ in autopsy series. Most hepatic hemangiomas are asymptomatic and are found incidentally in imaging studies. They are more frequent in women (3:1) and usually diagnosed between the ages of 30 and 50 years. These lesions are frequently solitary but may present as multiple lesions in one or both lobes of the liver. Most hepatic hemangiomas are less than $4 \mathrm{~cm}$ in diameter; those larger than $4 \mathrm{~cm}$ are known as giant hemangiomas. ${ }^{2}$ Giant hemangioma may present with abdominal pain or fullness, nausea, hemorrhage with resulting hemodynamic compromise, jaundice due to compression of the biliary tree, Budd-Chiari syndrome due to compression of hepatic veins, ${ }^{3}$ or as consumptive coagulopathy (also known as Kasabach- Merritt syndrome).

Pulmonary emboli usually originate from deep vein thrombosis (DVT) of lower extremity veins, but they may occasionally arise from DVT in less common locations, including the IVC. Thrombosis of the IVC is not rare but is usually under recognized. ${ }^{4} \mathrm{Hy}-$ percoagulability related to hematological or neoplas- tic abnormalities, venous stasis secondary to external compression of IVC by a neighboring pathologic process and vessel injury due to trauma have all been implicated as primary mechanisms in the pathophysiology of IVC thrombosis.

The distinctive feature of this case was an unusual presentation of a giant hepatic hemangioma causing IVC thrombosis leading to acute pulmonary embolism. There are few case reports of giant cavernous hemangioma presenting with acute pulmonary embolism in the literature. In one of the earlier reports, a 19-year-old man with severe right upper quadrant abdominal and back pain of 10 days duration suddenly developed shortness of breath and died soon afterward. Bilateral massive pulmonary thromboembolism with widespread thrombosis in a hepatic cavernous hemangioma was found at autopsy. In another report, a 35-year-old man who had acute pulmonary embolism was found to have IVC thrombosis, apparently resulting from compression of IVC by a hepatic hemangioma. ${ }^{7}$ There are two postulated mechanisms for the development of IVC thrombosis in the presence of giant hepatic hemangioma. The first mechanism is the development of new thrombosis in IVC due to sluggish flow induced by selective compression of 
IVC by a giant hepatic hemangioma, and the second is the migration of thrombi from the hemangioma into the hepatic veins and then into the IVC. Although partial thrombotic changes within the hepatic hemangiomas were seen on the delayed imaging in our case, the thrombi in the IVC was most likely caused by IVC compression by giant hemangiomas since the location of the thrombus in the retrohepatic IVC was more distal to where major hepatic veins drain to the IVC.

Most of the hemangiomas can be managed with close follow-up with no intervention. Treatment indications for giant hepatic hemangiomas are severe symptoms, complications, and inability to exclude malignancy. ${ }^{1}$ Surgical enucleation, resection, and transarterial catheter chemoembolization are the treatment options for single hemangiomas. Transplantation may be necessary in large unresectable lesions, multiple lesions, or those involving the hepatic hilum. Arkadopoulos et al reported successful surgical excision of a giant liver hemangioma that had caused pulmonary thromboembolism. ${ }^{8}$ Surgical excision was performed under inflow and outflow exclusion of the liver to prevent intraoperative migration of the intratumoral thrombi into the systemic circulation. In our patient, surgical resection was not feasible due to multisegmental involvement of the tumor; therefore long-term anticoagulation with warfarin was initiated. Liver transplantation referral was also made.

In conclusion, giant hepatic hemangiomas may cause IVC thrombosis, which can cause symptomatic pulmonary embolism. This case report highlights the importance of recognizing IVC thrombosis caused by intraabdominal pathologies in acute pulmonary emboli patients presenting with abnormal abdominal symptoms and findings.
Author Affiliation: Hatice Duygu Bas, Supannee Rassameehiran, Kazim Baser, and Weeraporn Srisung are residents in Internal Medicine at Texas Tech University Health Sciences Center in Lubbock, TX. Mamoun Bashir is a nephrologist, and Tinsay Woreta is a gastroenterologist in the Department of Internal Medicine at TTUHSC in Luubock, TX.

Received: 05/15/2016

Accepted: 05/24/2016

Reviewers: Cynthia Jumper MD

Published electronically: 07/15/2016

Conflict of Interest Disclosures: none

\section{REFERENCES}

1. Choi BY, Nguyen MH. The diagnosis and management of benign hepatic tumors. J Clinical Gastroenterology 2005; 39(5): 401-12.

2. Adam YG, Huvos AG, Fortner JG. Giant hemangiomas of the liver. Annals Surgery 1970; 172(2): 239-45.

3. Kim DY, Pantelic MV, Yoshida A, Jerius J, Abouljoud MS. Cavernous hemangioma presenting as Budd-Chiari syndrome. $J$ American College of Surgeons 2005; 200(3): 470-1.

4. Stein PD, Matta F, Yaekoub AY. Incidence of vena cava thrombosis in the United States. The American J Cardiology 2008; 102(7): 927-9.

5. Linnemann B, Schmidt H, Schindewolf M, et al. Etiology and VTE risk factor distribution in patients with inferior vena cava thrombosis. Thrombosis Research 2008; 123(1): 72-8.

6. Dennis PM. Fatal pulmonary embolism due to thrombosis of a hepatic cavernous haemangioma. Medicine, Science, Law 1980; 20(4): 287-8.

7. Paolillo V, Sicuro M, Nejrotti A, Rizzetto M, Casaccia M. Pulmonary embolism due to compression of the inferior vena cava by a hepatic hemangioma. Texas Heart Institute J / from the Texas Heart Institute of St Luke's Episcopal Hospital, Texas Children's Hospital 1993; 20(1): 66-8.

8. Arkadopoulos N, Stafyla V, Karapanos K, et al. Recurrent pulmonary embolism due to giant hepatic hamangioma treated with hepatectomy under vascular exclusion. Annals Vascular Surgery 2010; 24(6): 827 e1-3. 